of-function in the transcriptional expression of genes related to the NOTCH signalling pathway. The screening results were validated using TaqMan-based qPCR.

Results Treatment of HUVEC with the pro-angiogenic stimuli VEGF, bFGF, HGF, or HB-EGF induced in transient phosphorylation (activation) of ATF2 at Thr69 and Thr71. Suppression of ATF2 function by overexpression of ATF2AA in endothelial cells strongly attenuated VEGF- and bFGF-induced tubular morphogenesis. Loss of ATF2 function resulted in a significant increase in the VEGF- and bFGF-induced upregulation of Notch ligands DLL1 and DLL4 suggesting ATF2 involvement in the upregulation of these proteins.

Conclusion Our study demonstrates that growth factor-induced phosphorylation of ATF2 in residues 69 and 71 is essential for proper angiogenesis. Moreover, our results suggest that ATF2 plays a fundamental role in the regulation of Notch signalling-induced angiogenesis in endothelial cells.

Conflict of interest None

\section{BS46 HIGH CONTENT HIGH RESOLUTION CONFOCAL IMAGING TO CHARACTERISE MUTATIONS IN THE APELIN RECEPTOR IDENTIFIED IN PATIENTS FROM THE 100,000 GENOMES PROJECT}

${ }^{1}$ Tom Williams, 'Elisabeth O'Flaherty, ${ }^{1}$ Duuamene Nyimanu, ${ }^{1}$ Robyn Macrae, ${ }^{2}$ Heather Currinn, ${ }^{2} J a s o n$ Brown, ${ }^{2}$ Alastair Brown, ${ }^{1}$ Gregory Strachan, ${ }^{1}$ Stefan Gräf, ${ }^{10}$ Nicholas Morrell, ${ }^{1}$ Janet Maguire, ${ }^{1}$ Anthony Davenport, ${ }^{1}$ Rhoda E. Kuc. ' University of Cambridge; ${ }^{2}$ Sosei Heptares

10.1136/heartjnl-2019-BCS.207

Introduction The apelin receptor is a class A GPCR that binds two endogenous peptide ligands, apelin and elabela/toddler, to regulate the cardiovascular system. Apelin peptide levels are downregulated in pulmonary arterial hypertension (PAH) but the receptor remains present and clinically targetable. The NIHR BioResource BRIDGE study, a prospective component of the Genomics England 100,000 Genomes Project, has performed case-control genomic analysis of $\sim 7423$ patients with rare cardiovascular diseases including $\mathrm{PAH}$, and has identified a number of mutations in the apelin receptor in this cohort. Of these, 11 were selected for further assessment using high content imaging, following previous saturation binding studies that showed mutational effects on receptor expression and ligand binding.

Methods The Opera PhenixTM High Content Screening System was used to generate high-throughput triple fluorescence confocal images in fixed $\mathrm{CHO}-\mathrm{K} 1$ cells transiently transfected with eGFP-tagged wild-type or mutant apelin receptor. Cells were stained with wheat germ agglutinin-AlexaFluor-594 and Hoechst 33342 as membrane and nuclear markers respectively. Harmony High Content Imaging and Analysis Software was used to empirically quantify fluorescence intensities of the eGFP-tagged apelin receptor in the cytoplasm and membrane of transfected cells.

Results Images showed high expression of wild-type receptor with some degree of co-localisation with the membrane stain (fig 1A; fig 2). Mutation 2 showed visibly lower total protein and reduced membrane expression (fig 1B; fig 2). However, mutations 6 and 9 (Fig $1 \mathrm{C}$ and 1E respectively) showed similar expression and no significant difference in membrane co-localisation compared to wild-type (fig 2). Mutation 8 showed a trend for reduced membrane co-localisation (fig 1D) compared to wild-type. Note that membrane co-localisation differences closely matched differences in overall eGFP signal. Future work will aim to use machine learning to accurately isolate cell populations that show definite
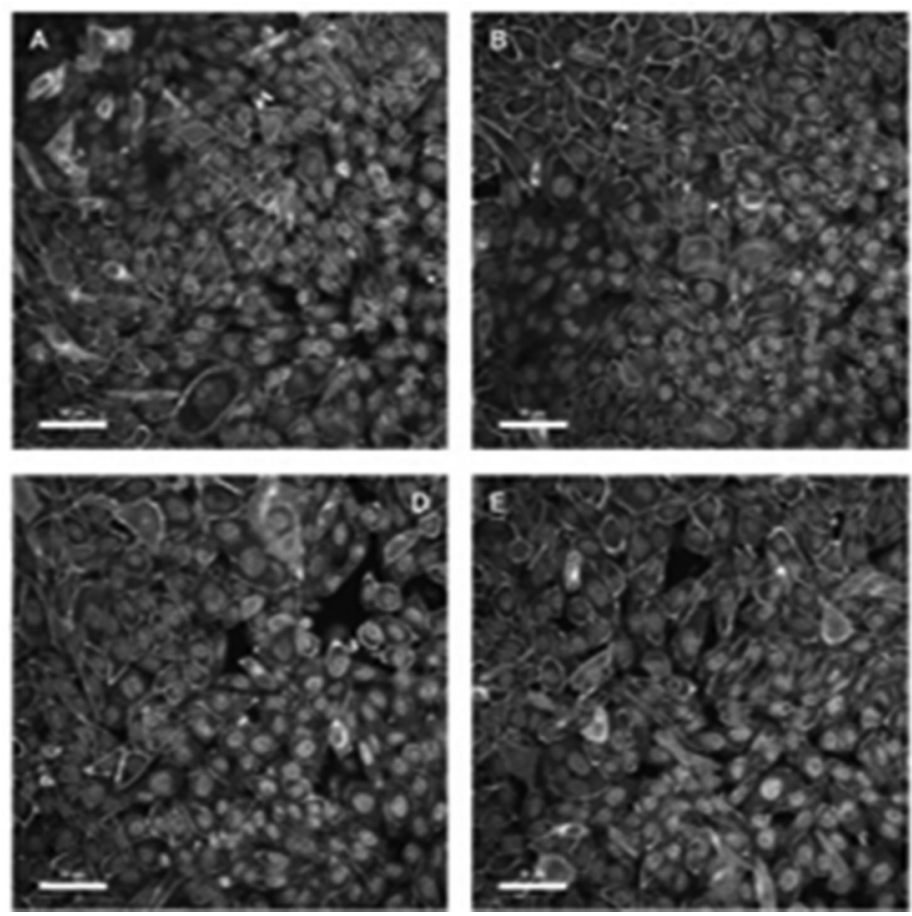

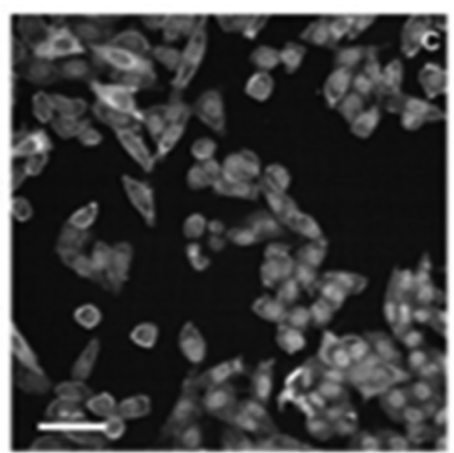

Fig 1: High contert triple fuorescence confocal images $\alpha$ CHOK1 cells transienty transtected with either: (A) wils. tope apelin receptor, (B) mutation 2. (C) muation 6. (D) muation 8 . of (E) mutation 9 . mages show eGFP taged receptor in green whea:germ agotion Af 594 conugate membrane stain in

orange, and Hoechas 30342 rucker stain in blue. Scale bar shows $50 \mathrm{um}$ 
apelin receptor-eGFP signal at the membrane before re-running analysis.

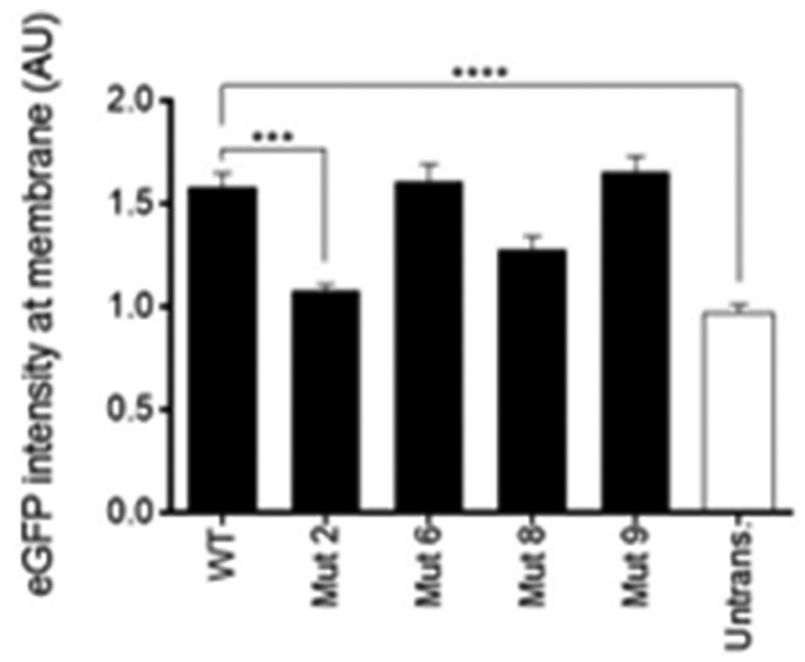

Abstract BS46 Figure 2

Conclusion We report the successful use of high content imaging to quantify differences in expression and membrane colocalisation of the apelin receptor when naturally occurring mutations identified in the NIHR BioResource BRIDGE study are introduced into the protein. When considered with receptor affinities and densities in previous saturation binding data, this work suggests that certain mutations may exhibit an overall impact on receptor expression (i.e. mutation 2), whilst others may directly alter ligand binding without significantly influencing protein levels (i.e. mutant 8). This work may provide great insight on the effects of real human apelin receptor mutations that may be contributing to disease phenotypes.

Conflict of interest none

\section{BS47 ROLE OF VASCULAR SMOOTH MUSCLE CELL-DERIVED EXOSOMES IN AGE-RELATED VASCULAR AMYLOIDOSIS}

Meredith Whitehead*, Sadia Ahmad, Catherine Shanahan. King's College London

\subsection{6/heartjnl-2019-BCS.208}

Background Ageing is a major risk factor for several vascular pathologies including calcification, the deposition of hydroxyapatite crystals in the vessel wall, and amyloid deposition. Exosomes, 20-100nm secreted vesicles, have been recognised as key mediators of amyloid formation in the brain and exosomes secreted from vascular smooth muscle cells (VSMCs) have been implicated in vascular calcification. However, whether exosomes link calcification and amyloid deposition in the vasculature is unknown.

Medin, forms the age-related aortic medial amyloid (AMA) and is present in the aorta of $97 \%$ of people over 50-years old. Medin is a 50 amino acid cleavage product of the protein MFGE8 however, the mechanism of AMA formation is unknown.

The aims of this study were to examine 1) changes in exosome secretion and content with age, 2) amyloid protein secretion and deposition by exosomes and 3) if calcifying conditions contribute to AMA formation.

Methods Western blotting, qPCR, and immunostaining were used to study medin and MFGE8 expression in primary human VSMCs, at different ages and in elevated calcium

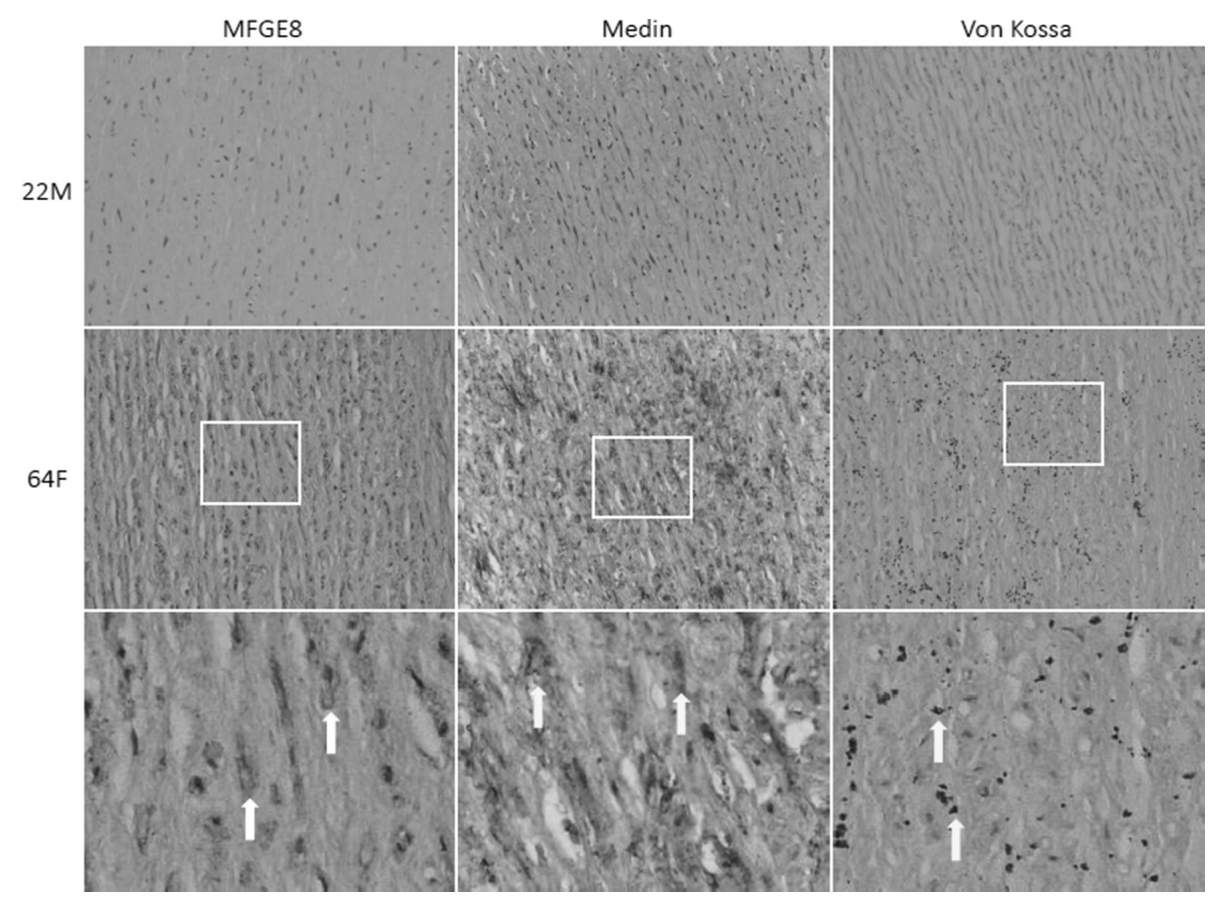

Abstract BS47 Figure 1 MFGE8, medin and calcification accumulate in aged aortas. Immunohistochemistry on aortas young (14-22 Years old) and ageing (54-78 years old) subjects. Amyloid precursor MFGE8 accumlates intracellularly in older subjects, indicated by the white arrows. Medin deposition appears mostly extracellular in the medial layer of aged aortas. Medial calcification is also observed by Von Kossa staining. The yellow arrows indicate areas of extracellular staining 